\title{
Coronal oblique T1 weighted magnetic resonance image of the sacroiliac joints
}

This Endgames quiz (BMJ 2015;350:h1306, doi:10.1136/bmj. h1306) incorrectly identifies structure E as the "left obturator internus muscle," when this is, in fact, the left piriformis muscle.
Cite this as: BMJ 2015;350:h1911

๑ B BMJ Publishing Group Ltd 2015 